Ciência Florestal, Santa Maria, v. 24, n. 2, p. 501-507, abr.-jun., 2014

ISSN 0103-9954

\title{
CONTRIBUIÇÃO DOS POLEIROS ARTIFICIAIS NA DISPERSÃO DE SEMENTES E SUA APLICAÇÃO NA RESTAURAÇÃO FLORESTAL
}

\author{
CONTRIBUTION OF ARTIFICIAL PERCHES TO SEED DISPERSAL AND ITS APPLICATION TO \\ FOREST RESTORATION
}

\author{
Cristiano Roberto Dias ${ }^{1}$ Fabiana Umetsu ${ }^{2}$ Tiago Böer Breier ${ }^{3}$
}

\begin{abstract}
RESUMO
O uso de poleiros artificiais é uma técnica eficiente para a restauração florestal. Os objetivos desse trabalho foram: (1) avaliar o efeito da presença de poleiros artificiais no número de sementes florestais depositadas em coletores; (2) identificar as síndromes de dispersão das sementes, e (3) analisar o efeito da distância da borda da floresta no aporte de sementes. As atividades de campo foram realizadas no município de Iguaba Grande, Rio de Janeiro, Brasil. Foram instalados 70 coletores de sementes (com 0,50 m de diâmetro) distribuídos em transectos distantes paralelamente 5,15 e $35 \mathrm{~m}$ da borda da floresta em área de pasto abandonado e em um transecto de $10 \mathrm{~m}$ no interior da floresta. Cada transecto fora do fragmento recebeu dez coletores, instalados sob poleiros artificiais, e dez coletores, instalados sem poleiro. No interior do fragmento foram instalados dez coletores, sem presença de poleiro artificial. Durante três semanas de amostragem foram coletadas 418 sementes, sendo 242 zoocóricas $(57,9 \%)$ e 176 anemocóricas $(42,1 \%)$. A média de sementes com presença de poleiro artificial foi de $7,4( \pm 3,9)$ sementes $/ \mathrm{m}^{2} / \mathrm{mês}$, enquanto na ausência de poleiro artificial, a média foi de foi de $1,7( \pm 1,8)$ sementes $/ \mathrm{m}^{2} / \mathrm{mês}$, considerando simultaneamente sementes anemocóricas e zoocóricas. Diferentes distâncias da borda da floresta, até $35 \mathrm{~m}$, não influenciaram no aporte de sementes zoocóricas e anemocóricas. A presença de poleiros artificiais levou a um aporte 118 vezes maior de sementes zoocóricas quando comparada aos coletores sem poleiros. Poleiros artificiais podem funcionar como estrutura catalisadora da restauração florestal, com significativo incremento no aporte de sementes zoocóricas.
\end{abstract}

Palavras-chave: chuva de sementes; zoocoria; anemocoria; sucessão; regeneração florestal.

\section{ABSTRACT}

The use of artificial perches is an efficient technique to forest restoration. We aimed to (1) evaluate the effect of the presence of artificial perches on the number of forest seeds deposited on seed fall collectors, (2) identify the seed dispersal syndromes, and (3) to analyze the effect of distance from the forest border on seed input. Field work was performed in the municipality of Iguaba Grande, Rio de Janeiro, Brazil. We installed 70 seed fall collectors (with diameter of $0.50 \mathrm{~m}$ ) distributed in transects parallelly distant 5,15 e $35 \mathrm{~m}$ from the forest border in abandoned pasture, and in one transect $10 \mathrm{~m}$ from the border, in the forest interior. Each transect outside the forest patch received ten seed fall collectors, installed under artificial perches, and ten seed fall collectors, installed without perches. In the forest interior, we installed ten collectors. During three weeks of sampling, we obtained 418 seeds, classified into 242 zoochoric seeds (57.9\%) and 176 anemochoric seeds (42.1\%). The average seed with the presence of artificial perch was 7.4 $( \pm 3.9)$ seeds $/ \mathrm{m}^{2} /$ month while in the absence of artificial perch the average was $1.7( \pm 1.8) \mathrm{seeds} / \mathrm{m}^{2} / \mathrm{month}$, considering anemochoric and zoochoric seeds. Different distances from the forest edge, up to $35 \mathrm{~m}$, did not

1 Engenheiro Florestal, Técnico do Núcleo Experimental de Iguaba Grande, Universidade Federal Fluminense, Rodovia Amaral Peixoto, Km 100, CEP 28960-000, Iguaba Grande (RJ), Brasil. cdias_floresta@yahoo.com.br

2 Bióloga, Pós-Doutoranda do Programa de Pós-Graduação em Engenharia Civil da COPPE, Universidade Federal do Rio de Janeiro, Av. Horácio Macedo 2030, CEP 21945-970, Rio de Janeiro (RJ), Brasil. fabiume@yahoo.com

3 Biólogo, Dr., Professor Adjunto do Laboratório de Biologia Reprodutiva e Conservação de Espécies Arbóreas, Universidade Federal Rural do Rio de Janeiro, Rodovia BR 465, Km 07, CEP 23890-000, Seropédica (RJ), Brasil. tiagobreier@gmail.com

Recebido para publicação em 23/03/2012 e aceito em 22/03/2013 
influence the number of zoochoric and anemochoric seeds. The presence of artificial perches increased at 118 times the number of seeds dispersed by animals when compared to traps without perches. Artificial perches work as catalyzing structures for forest restoration, with a significant increase in the input of zoochoric seeds.

Keywords: seed rain; zoochory; anemochory; succession; regeneration.

\section{INTRODUÇÃO}

Extensas áreas florestais nos trópicos foram e estão sendo convertidas em pastagens para criação de gado e, frequentemente, quando abandonadas, perdem sua resiliência, de modo que retarda ou inviabiliza o processo de sucessão natural necessário para a regeneração da cobertura florestal. Entre os motivos da perda da resiliência da floresta estão: distância da fonte de sementes, ausência de dispersores, tamanho dos diásporos, predação de sementes, competição com gramíneas, ocorrência de incêndios, limitações microclimáticas e de solo, as quais influenciam no crescimento das plantas e perda do banco de sementes nativas outrora presente no solo (AIDE e CAVELIER, 1994; WUNDERLE, 1997; GRISCOM et al., 2009).

Considerando a dificuldade da chegada de diásporos zoocóricos em áreas de pastagens abandonadas, a presença de poleiros pode contribuir para o incremento no aporte de sementes. Poleiros vivos ou naturais apresentam uma importante contribuição no incremento do aporte de sementes zoocóricas em meio a áreas abertas e são preferidos pela fauna dispersora quando comparados a poleiros secos ou artificiais (SANTOS e PILLAR, 2007). Contudo, as diferentes espécies arbóreas, quando isoladas na pastagem, apresentam diferentes graus de visitação pela fauna, o que está relacionado, principalmente, com o tipo de dispersão (biótica ou abiótica) e à arquitetura da copa da espécie arbórea (SLOCUM e HORVITZ, 2000). Estudos realizados com árvores frutíferas nativas da Mata Atlântica detectaram a atração de muitas espécies de aves frugívoras, como em um indivíduo da espécie arbórea Alchornea glandulosa Poepp. (Euphorbiaceae) onde foram registradas 14 espécies de aves frugívoras em 20 horas de observação (VALENTE, 2001). E em um indivíduo da espécie arbórea Tapirira guianensis Aubl. (Anacardidaceae) onde foram registradas 15 espécies de aves frugívoras em 56 horas de observação durante a fase de dispersão dos frutos (GUIMARÃES, 2003).

A estrutura do poleiro também pode influenciar na frequência de uso pela fauna. Em um estudo realizado na Costa Rica, foram instalados dois tipos poleiros artificiais em pastagens abandonadas próximas a remanescentes florestais. Os coletores sem poleiro apresentaram apenas duas espécies de sementes zoocóricas, enquanto os coletores localizados abaixo dos poleiros secos feitos com barras horizontais de madeira apresentaram seis espécies de sementes, e os coletores localizados abaixo dos poleiros feitos de galhos de árvores apresentaram 13 espécies (HOLL, 1998).

Em áreas de pasto abandonado, fatores como a presença de poleiros, a presença de estrutura complexa da vegetação e de recursos alimentares podem atrair a fauna com maior intensidade (WUNDERLE, 1997). Em estudo realizado sobre a influência da estrutura da vegetação na riqueza de aves frugívoras em área Amazônia Oriental detectou que, das 47 espécies de aves frugívoras registradas em área de floresta secundária, apenas 18 frequentavam áreas adjacentes de pastagem abandonada com presença de arbustos. E somente três espécies frequentavam áreas de pastagem com forrageamento constante pelo gado (SILVA et al., 1995).

Além das aves frugívoras, outros vertebrados alados como morcegos frugívoros podem contribuir para a dispersão de sementes. Em trabalho realizado em área de floresta em regeneração nas Filipinas comparando dispersão de sementes florestais por aves e morcegos, com uso de pares de coletores noturnos e diurnos, detectou que as aves foram os dispersores mais importantes nesta área de estudo e sugere que esforços de restauração devem focar na atração destes dispersores para áreas degradadas (GONZALES et al., 2009). Contudo, trabalhos realizados no México detectaram que os morcegos dispersam maior quantidade de sementes e maior quantidade de espécies do que as aves (MEDELÍN e GAONA, 1999) e que existem nítidas diferenças entre algumas espécies de plantas dispersadas no período noturno e no período diurno (GALINDOGONZÁLEZ et al., 2000).

O objetivo desse trabalho foi avaliar o efeito da presença de poleiros artificiais no número 
de sementes florestais depositadas em coletores, identificar as síndromes de dispersão das sementes, e analisar o efeito da distância à borda da floresta no aporte de sementes.

\section{MATERIAL E MÉTODOS}

O trabalho foi desenvolvido na Área de Proteção Ambiental Morro do Governo, município Iguaba Grande, Rio de Janeiro, coordenadas $22^{\circ} 50^{\prime} 55^{\prime}$ 'S e $42^{\circ} 11^{\prime} 58^{\prime \prime} \mathrm{W}$. A vegetação original é a floresta de restinga, sendo que o experimento foi desenvolvido em borda de floresta de restinga e área adjacente composta por pasto abandonado com predomínio de gramíneas. A estação meteorológica mais próxima da área de estudo é a de Cabo Frio, onde são registrados $770,9 \mathrm{~mm}$ de precipitação anual média e temperatura média anual de $23,6^{\circ} \mathrm{C}$ (BRASIL, 1992).

Os solos da região são eutróficos, considerando o baixo grau de intemperismo e a presença de minerais primários com reserva de nutrientes. Estes solos são influenciados pelo clima da região, que é mais seco em relação a outras regiões do estado do Rio de Janeiro, o que condiciona um ambiente peculiar com forte tendência à salinização e xerofitismo (IBRAIMO et al., 2004). A área de estudo é de interesse conservacionista, pois representa o último reduto expressivo da floresta de restinga das margens continentais dos lagos da Região dos Lagos no Rio de Janeiro.

Os poleiros artificiais (poleiros secos) foram construídos com uso de bambus com $2 \mathrm{~m}$ de altura e com estruturas transversais para o pouso das aves, sendo uma das estruturas composta por duas varas perpendiculares com $50 \mathrm{~cm}$ cada, e outra estrutura com duas varas perpendiculares de $100 \mathrm{~cm}$ cada, colocadas a $1,70 \mathrm{~cm}$ e $1,10 \mathrm{~cm}$ do solo, respectivamente.

Os coletores com $50 \mathrm{~cm}$ de diâmetro foram confeccionados com sacos plásticos resistentes com pequenos orifícios para drenagem de água e colocados a $50 \mathrm{~cm}$ acima do solo. A amostragem foi realizada durante um período de três semanas em julho de 2008. Ao todo foram instalados 70 coletores, sendo 30 coletores instalados debaixo dos poleiros artificiais, 30 coletores controle (sem poleiros artificiais) e dez coletores foram instalados no interior da floresta.

Os coletores com poleiros artificiais e coletores sem poleiros foram dispostos em três transectos distribuídos na área de pasto abandonado e distantes paralelamente 5,15 e $35 \mathrm{~m}$ da borda da floresta. Os coletores com poleiro artificial e sem poleiro artificial foram intercalados ao longo do mesmo transecto. Um quarto transecto de coletores sem poleiros artificiais foi instalado no interior da floresta, a $10 \mathrm{~m}$ da borda. A distância entre coletores foi de $3 \mathrm{~m}$.

As sementes foram triadas no laboratório da Universidade Federal Fluminense, separadas em morfoespécies, e classificadas de acordo com a síndrome de dispersão (PIJL, 1972), considerando o sua morfologia. Para testar o efeito da distância em relação à borda da floresta no aporte de sementes nos coletores, e fazer a comparação entre presença e ausência de poleiros, foi avaliada a normalidade com o teste de Lilliefors e a comparações entre médias com o teste Kruskal-Wallis pelo método de Dunn, calculado com o Programa BioEstat 5.0.

\section{RESULTADOS E DISCUSSÃO}

$\mathrm{Na}$ amostragem realizada nos 70 coletores instalados durante três semanas (21 dias), foram coletadas 418 sementes, sendo 242 zoocóricas $(57,9 \%)$ e 176 anemocóricas (42,1\%). Não foram encontradas sementes barocóricas e sementes autocóricas. Os resultados detalhados são apresentados na Tabela 1.

Os poleiros artificiais apresentaram contribuição significativa no aporte de sementes florestais na área de pasto abandonado, sendo que o número absoluto de sementes amostradas na presença de poleiro artificial (305) foi maior que na ausência de poleiro (70) o que equivale a $222 \mathrm{sementes} / \mathrm{m}^{2} /$ mês na presença de poleiro e 50 sementes $/ \mathrm{m}^{2} / \mathrm{mês}$ na ausência de poleiro. Esse número se deve principalmente à presença de sementes zoocóricas. Assim, o uso de poleiros artificiais figura como uma técnica eficiente para o incremento do aporte de sementes quando instalados próximos à fonte de sementes, e têm sido indicados por diversos autores como uma importante ferramenta para a restauração florestal no Brasil e outros países da América Latina (AIDE e CAVELIER, 1994; SHIELS e WALKER, 2003; ZANINI e GANADE, 2005; REIS et al., 2007; BECHARA et al., 2007; REGENSBURGER et al., 2008; RODRIGUES et al., 2009).

$\mathrm{Na}$ área de pasto abandonado, a média cumulativa de sementes, nos coletores com poleiro artificial, foi de 7,4 $( \pm 3,9)$ sementes $/ \mathrm{m}^{2} /$ mês, enquanto nos coletores sem poleiro, foi de 
TABELA 1: Número absoluto de sementes amostradas nos coletores durante 21 dias e estimativa do número de sementes $/ \mathrm{m}^{2} /$ mês (valores entre parêntesis) de acordo com a síndrome de dispersão, transecto e presença/ausência de poleiros artificiais, município de Iguaba Grande, Rio de Janeiro, Brasil.

TABLE 1: Absolute number of seeds sampled in the traps in 21 days and estimated number of seeds $/ \mathrm{m}^{2} /$ month (values in parentheses) according to the dispersion syndrome, transect and presence / absence of artificial perches, Iguaba Grande, Rio de Janeiro state, Brazil .

\begin{tabular}{cccccccc}
\hline $\begin{array}{c}\text { Síndrome de } \\
\text { dispersão }\end{array}$ & $\begin{array}{c}\text { Interior da } \\
\text { floresta } \\
(-10 \mathrm{~m})\end{array}$ & $\begin{array}{c}\text { Presença } \\
\text { de poleiro } \\
\text { artificial } \\
(5 \mathrm{~m})\end{array}$ & $\begin{array}{c}\text { Ausência } \\
\text { de poleiro } \\
\text { artificial } \\
(5 \mathrm{~m})\end{array}$ & $\begin{array}{c}\text { Presença } \\
\text { de poleiro } \\
\text { artificial } \\
(15 \mathrm{~m})\end{array}$ & $\begin{array}{c}\text { Ausência } \\
\text { de poleiro } \\
\text { artificial } \\
(15 \mathrm{~m})\end{array}$ & $\begin{array}{c}\text { Presença } \\
\text { de poleiro } \\
\text { artificial } \\
(35 \mathrm{~m})\end{array}$ & $\begin{array}{c}\text { Ausencia } \\
\text { de poleiro } \\
\text { artificial } \\
(35 \mathrm{~m})\end{array}$ \\
\hline Anemocoria & $39(28)$ & $24(17)$ & $39(28)$ & $31(73)$ & $18(13)$ & $15(11)$ & $10(7)$ \\
Zoocoria & $4(3)$ & $78(57)$ & $1(1)$ & $100(23)$ & $1(1)$ & $57(41)$ & $0(0)$ \\
\hline Total & $43(31)$ & $102(74)$ & $40(29)$ & $131(96)$ & $20(14)$ & $72(52)$ & $10(7)$ \\
\hline
\end{tabular}

$1,7 \quad( \pm 1,8) \quad$ sementes $/ \mathrm{m}^{2} / \mathrm{mês}, \quad$ considerando anemocóricas e zoocóricas. Para sementes anemocóricas, a média cumulativa com poleiro artificial foi de $1,7( \pm 1,4)$ sementes $/ \mathrm{m}^{2} /$ mês, e nos coletores sem poleiro, foi de $1,6( \pm 1,8)$ sementes/ $\mathrm{m}^{2} / \mathrm{mês}$, ou seja, a presença de poleiro não interfere no aporte de sementes anemocóricas. Para sementes zoocóricas, a média cumulativa com poleiro artificial foi de $5,7( \pm 3,4)$ sementes $/ \mathrm{m}^{2} /$ mês, e nos coletores sem poleiro, foi de $0,1( \pm 0,3)$.

Considerando o aporte quantitativo de sementes anemocóricas no interior da floresta e a diferentes distâncias da borda $(5,15$ e $35 \mathrm{~m})$ fica evidente que as sementes anemocóricas não respondem à presença ou ausência de poleiros e que sua distribuição não é influenciada, até $35 \mathrm{~m}$, pela distância da borda da floresta (Figura 1). A única diferença detectada foi na comparação entre os coletores localizados no interior da floresta e os coletores dispostos a $35 \mathrm{~m}$ da borda sem poleiro $(\mathrm{p}<0,05)$.

O padrão encontrado onde o número de sementes anemocóricas não foi influenciado pela distância da borda da floresta até $35 \mathrm{~m}$ difere dos resultados descritos para Colômbia, onde a chuva de sementes decresceu rapidamente do interior da floresta para o interior da pastagem, até $20 \mathrm{~m}$, considerando sementes zoocóricas e anemocóricas simultaneamente e uso de poleiros artificiais (AIDE e CAVELIER, 1994). E nas Filipinas, onde a dispersão de sementes anemocóricas ou sementes não dispersas por vertebrados foi maior no interior da floresta, do que a $40 \mathrm{~m}$ de distância da borda em meio a uma área em regeneração (INGLE, 2003).

Com relação a sementes zoocóricas, na Tabela 1, o número absoluto amostrado nos coletores com presença de poleiro a diferentes distâncias da borda $(78,100$ e 57$)$ foi expressivamente maior que na ausência de poleiros $(1,1,0)$ e no interior da floresta (4). Considerando apenas os transectos dispostos na área de pasto abandonado, os diásporos zoocóricos totalizam 237 unidades, sendo que os coletores que receberam os poleiros artificiais apresentaram o maior percentual deste aporte, com 235 diásporos $(99,2 \%)$, contra apenas dois diásporos nos coletores controle $(0,8 \%)$. A presença de poleiros artificiais levou a um aporte 118 vezes maior de sementes zoocóricas quando comparada aos coletores sem poleiros.

$\mathrm{Na}$ Figura 2 fica evidente a contribuição dos poleiros artificiais no aporte quantitativo de sementes zoocóricas sendo que os tratamentos sem poleiro formam um grupo com reduzido ou nulo no aporte de sementes zoocóricas $(\mathrm{p}<0,05)$. Os tratamentos com presença de poleiro artificial a diferentes distâncias da borda não diferem estatisticamente entre si $(\mathrm{p}<0,05)$ indicando que para as aves dispersoras poleiros distribuídos em áreas de pasto abandonado a distâncias de até $35 \mathrm{~m}$ da borda da floresta são indiferentes e igualmente frequentados. Desta forma, recomendam-se estudos com o objetivo de elucidar qual a distância máxima de alcance da técnica de poleiros artificiais na restauração florestal nos diferentes biomas brasileiros.

Ao todo foram coletadas sete morfoespécies de sementes florestais. A principal espécie anemocórica encontrada foi uma Fabaceae: Pterogyne nitens Tul. (amendoim-bravo), que é uma pioneira arbórea e representou cerca de $98 \%$ das sementes anemocóricas. Este fato está relacionado com a abundância desta espécie na área e o período de 


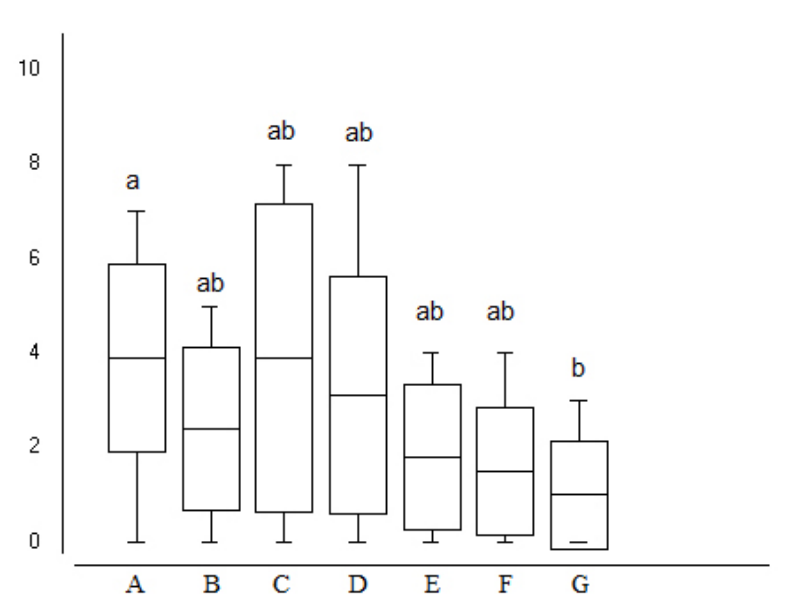

FIGURA 1: Média, desvio padrão, valores máximo e mínimo de sementes anemocóricas coletadas, com cada coluna representando dez coletores. A- interior da floresta. B- presença de poleiro a $5 \mathrm{~m}$ da borda. C- ausência de poleiro a $5 \mathrm{~m}$ da borda. D- presença de poleiro a $15 \mathrm{~m}$ da borda. E- ausência de poleiro a $15 \mathrm{~m}$ da borda. F- presença de poleiro a $35 \mathrm{~m}$ da borda. G- ausência de poleiro a $35 \mathrm{~m}$ da borda. Letras distintas significam diferenças detectadas pelo teste de Kruskal-Wallis a 5\%.

FIGURE 1: Mean, standard deviation, maximum and minimum values of anemochorical seeds collected, each column represents ten collectors. A- forest interior. Bpresence of perch at $5 \mathrm{~m}$ from the border. C- absence of perch at $5 \mathrm{~m}$ from the border. D- presence of perch at $15 \mathrm{~m}$ from the border. E- absence of perch at $15 \mathrm{~m}$ from the border. F- presence of perch at $35 \mathrm{~m}$ from the border. G- absence of perch at $35 \mathrm{~m}$ from the border. Different letters mean differences detected by Mann-Whitney test at $5 \%$.

amostragem coincidir com a maturação e dispersão desta espécie. Entre as sementes zoocóricas, uma Myrtaceae: Siphoneugena guilfoyleiana Proença (vamirim-de-ferro) foi a espécie mais abundante, com $74 \%$ das sementes zoocóricas, e as demais sementes zoocóricas pertenceram a outras quatro morfoespécies distintas.

As cinco morfoespécies de sementes zoocóricas encontradas no presente estudo refletem a

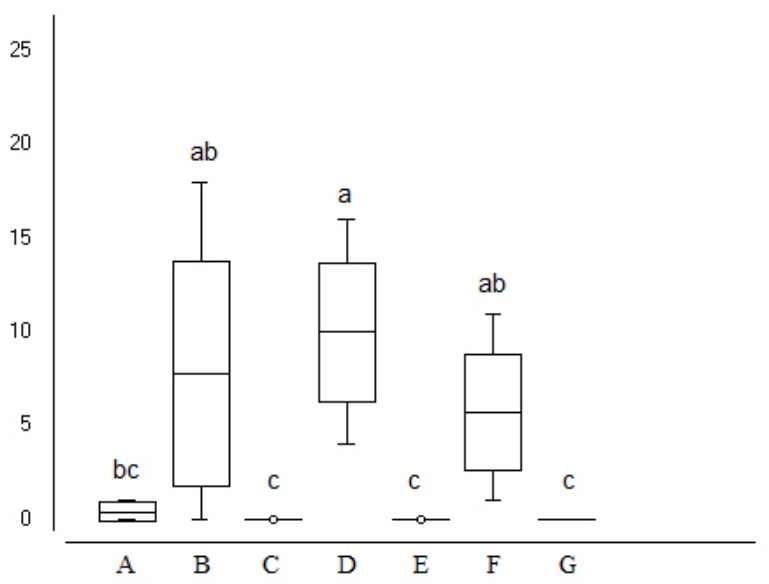

FIGURA 2: Média, desvio padrão, valores máximo e mínimo de sementes zoocóricas coletadas, com cada coluna representando dez coletores. A- interior da floresta. B- presença de poleiro a $5 \mathrm{~m}$ da borda. C- ausência de poleiro a $5 \mathrm{~m}$ da borda. D- presença de poleiro a $15 \mathrm{~m}$ da borda. E- ausência de poleiro a $15 \mathrm{~m}$ da borda. F- presença de poleiro a $35 \mathrm{~m}$ da borda. G- ausência de poleiro a $35 \mathrm{~m}$ da borda. Letras distintas significam diferenças detectadas pelo teste de Kruskal-Wallis a 5\%.

FIGURE 2: Mean, standard deviation, maximum and minimum values of zoochorical seeds collected, each column represents ten collectors. A- forest interior. B- presence of perch at $5 \mathrm{~m}$ from the border. $\mathrm{C}$ - absence of perch at $5 \mathrm{~m}$ from the border. D- presence of perch at $15 \mathrm{~m}$ from the border. E- absence of perch at $15 \mathrm{~m}$ from the border. F- presence of perch at $35 \mathrm{~m}$ from the border. G- absence of perch at $35 \mathrm{~m}$ from the border. Different letters mean differences detected by Mann-Whitney test at 5\%.

quantidade de espécies que estavam sendo dispersas no período de duração dos trabalhos de campo, ou seja, três semanas, e este resultado não reflete a quantidade de espécies com sementes zoocóricas do fragmento por se tratar de uma amostragem com período determinado e inferior a um ciclo anual. Silva et al. (2009) em trabalho realizado em floresta de restinga da Ilha Comprida (SP) com coletores distribuídos dentro da floresta ao longo de 19 meses 
de observação registraram $72,7 \%$ de sementes zoocóricas e 27,3\% de sementes anemocóricas. Esses dados contrastam com o presente trabalho onde, considerando os coletores distribuídos dentro da floresta, foram encontrados 9,5\% (4) de sementes zoocóricas e 90,5\% (38) de sementes anemocóricas.

\section{CONCLUSÕES}

O uso de poleiros artificiais foi eficiente no incremento do aporte de sementes florestais zoocóricas em áreas de pastagem abandonada, quando instalados próximos à fonte de sementes, mesmo em um curto período de observação. Técnicas como esta podem contribuir na redução de custos e também no planejamento de ações de restauração florestal na escala da paisagem. Contudo, futuros estudos fazem-se necessários para a mensuração do alcance da técnica em distâncias superiores a $35 \mathrm{~m}$ da borda da floresta, de acordo com o tipo florestal, na avaliação do formato e altura do poleiro, no desenvolvimento de métodos que favoreçam a germinação das sementes e estabelecimento das mudas nas áreas de restauração.

\section{REFERÊNCIAS BIBLIOGRÁFICAS}

AIDE, T. M.; CAVELIER, J. Barriers to lowland tropical forest restoration in the Sierra Nevada de Santa Marta, Colombia. Restoration Ecology, Washington D. C., v. 2, p. 219-229. 1994.

BECHARA, F. C. et al. Unidades Demonstrativas de Restauração Ecológica através de Técnicas Nucleadoras de Biodiversidade. Revista Brasileira de Biociências, Porto Alegre, v. 5, p. 9-11, 2007. BRASIL. Ministério da Agricultura e Reforma Agrária. Normais climatológicas (1961-1990). Brasília: Ministério da Agricultura e Reforma Agrária, $1992.84 \mathrm{p}$.

GALINDO-GONZÁLEZ, J. et al. Bat- and bird-generated seed rains at isolated trees in pastures in a tropical rainforest. Conservation Biology, Washington D.C., v. 14, n. 6, p. 1693-1699. 2000. GONZALES, R. S. et al. Seed dispersal by birds and bats in lowland Philippine forest successional area. Biotropica, Zurich, v. 41, n. 4, p. 452-458. 2009. GRISCOM, H. P. et al. Forest Regeneration from Pasture in the Dry Tropics of Panama: Effects of Cattle, Exotic Grass, and Forested Riparia. Restoration Ecology, Washington D. C., v. 17, p. 117-126. 2009.

GUIMARÃES, M. A. Frugivoria por aves em
Tapirira guianensis (Anacardiaceae) na zona urbana do município de Araruama, estado do Rio de Janeiro, sudeste brasileiro. Atualidades Ornitológicas, Ivaiporã, v. 16, p. 12. 2003.

HOLL, K. D. Do Bird Perching Structures Elevate Seed Rain and Seedling Establishment in Abandoned Tropical Pasture? Restoration Ecology, Washington D. C., v. 6, p. 253-261. 1998.

IBRAIMO, M. M. et al. Gênese e micromorfologia de solos sob vegetação xeromórfica (caatinga) na Região dos Lagos (RJ). Revista Brasileira de Ciência do Solo, Viçosa, v. 28, p. 695-712. 2004.

INGLE, N. R. Seed dispersal by wind, birds, and bats between Philippine montane rainforest and successional vegetation. Oecologia, Berlin, v. 134, p. 251-261. 2003.

MCCLANAHAN, T.R.; WOLFE, R.W.Accelerating forest succession in a fragmented landscape: the role of birds and perches. Conservation Biology, Washington D. C., v. 7, p. 279-288. 1993.

MEDELLÍN, R. A., GAONA, O. Seed dispersal by bats and birds in forest and distubed habitats of Chiapas, México. Biotropica, Marburg, v. 31, n. 3, p. 478-485. 1999.

MELO, V. A. et al. Efeito de poleiros artificiais na dispersão de sementes por aves. Revista Árvore, Viçosa, v. 24, p. 235-240. 2000.

PIJL, L. van der. Principles of dispersal in higher plants. Berlin: Springer, 1972. 161 p.

REGENSBURGER, B. et al. Integração de técnicas de solo, plantas e animais para recuperar áreas degradadas. Ciência Rural, Santa Maria, v. 38, p. 1773-1776. 2008.

REIS, A. et al. Restauração na Floresta Ombrófila Mista através da sucessão natural. Pesquisa Florestal Brasileira, Colombo, v. 55, p. 67-73. 2007.

RODRIGUES, R. R. et al. On the restoration of high diversity forests: 30 years of experience in the Brazilian Atlantic Forest. Biological Conservation, London, v. 142, n. 6, p. 1242-1251. 2009.

SANTOS, M. M. G.; PILLAR, V. D. Influência de Poleiros Naturais e Artificiais na Expansão da Floresta com Araucária sobre os Campos, em São Francisco de Paula, RS. Revista Brasileira de Biociências, Porto Alegre, v. 5, p. 594-596. 2007.

SILVA, C. R. et al. Chuva de sementes em uma floresta alta de restinga em Ilha Comprida (SP). Cerne, Lavras, v. 15, n. 3, p. 355-365. 2009.

SILVA, J. M. C. et al. Plant succession, landscape managemente, and the ecology of frugivorous 
birds in abandoned amazonian pastures. aves em Alchornea glandulosa (Euphorbiaceae) em Conservation Biology, Washington D. C., Rio Claro, São Paulo. Iheringia Série Zoológica, v. 10, n. 2, p. 491- 503. 2010.

Porto Alegre, v. 91, p. 61-66. 2001.

SHIELS, A. B.; WALKER, L. R. Bird Perches WUNDERLE, J. M. The role of animal seed Increase Forest Seeds on Puerto Rican Landslides. dispersal in accelerating native Forest regeneration Restoration Ecology, Washington D. C., v. 11, on degraded tropical lands. Forest Ecology p. 457-465. 2003.

SLOCUM M. G.; HORVITZ, C. C. Seed arrival under different genera of trees in a neotropical pasture. Plant Ecology, Amsterdam, v. 149, p. 51-62. 2000.

VALENTE, R. M. Comportamento alimentar de and Management, Amsterdam, v. 99, p. 223-235. 1997.

ZANINI, L.; GANADE, G. Restoration of Araucaria Forest: The Role of Perches, Pioneer Vegetation, and Soil Fertility. Restoration Ecology, Washington D. C., v. 13, p. 507-514. 2005. 